\title{
Characterization of inclined GaSb nanopillars by angle resolved Mueller polarimetry
}

\author{
I. S. Nerbø ${ }^{1}$, M. Foldyna ${ }^{2}$, M. Kildemo ${ }^{1}$, A. De Martino ${ }^{2}$, S. Le Roy ${ }^{3}$ and E. Søndergård ${ }^{1}$ \\ ${ }^{1}$ Department of Physics, Norwegian University of Science and Technology, NO-7491, Trondheim, Norway \\ ${ }^{2}$ LPICM, Ecole polytechnique, CNRS, 91128, Palaiseau, France \\ ${ }^{3}$ UMR 125 CNRS/Saint-Gobain, F-93303, Aubervilliers, France
}

\begin{abstract}
Color in living organisms is primarily generated by two mechanisms: selective absorption by pigments and structural coloration, or a combination of both. In this study, we investigated the coloration of cuticle from the wings (elytra) of the two ground beetle species Carabus auronitens and Carabus auratus. The greenish iridescent color of both species is created by a multilayer structure consisting of periodically alternating layers with different thicknesses and composition which is located in the 1-2 $\mu \mathrm{m}$ thick outermost layer of the cuticle (epicuticle). Illuminated with white light, reflectance spectra in both linear polarisation show an angle-dependent characteristic peak in the blue/green region of the spectrum. Furthermore, the reflected light is polarised linearly. Scattering experiments with laser illumination at $532 \mathrm{~nm}$ show diffuse scattering over a larger angular range. The polarisation dependence of the scattered light is consistent with the interpretation of small inhomogeneities as scattering centres in the elytra.
\end{abstract}

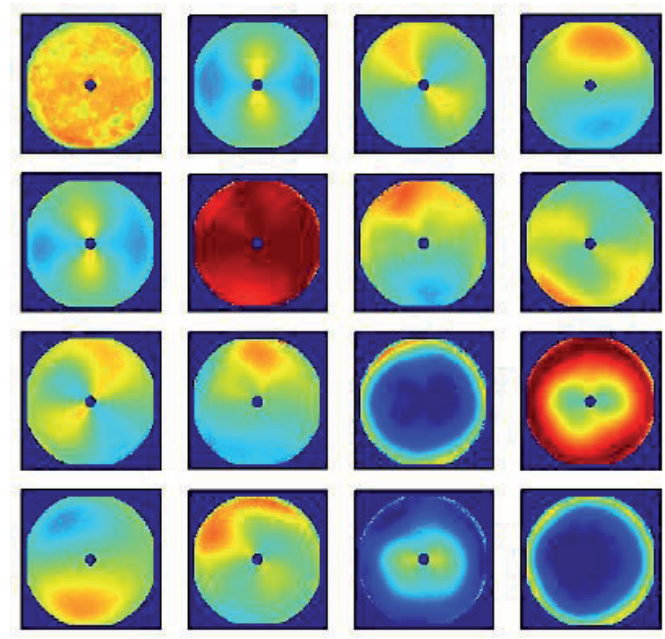

Figure. Polar plot of angle resolved Mueller matrix measurement of inclined GaSb nanopillars.

This is an Open Access article distributed under the terms of the Creative Commons Attribution-Noncommercial License 3.0, which permits unrestricted use, distribution, and reproduction in any noncommercial medium, provided the original work is properly cited. 